were analysed. Qualitative data was collected through a semi structured questionnaire and a Braun and Clarke (2006) method of thematic analysis was carried out.

Results The majority of patients found that TFU post cataract operation was an acceptable way to receive follow up. On average patients scored $4.94 / 5$ on the likert scale for the simplicity and ease of use. Overarching themes that emerged were that TFU is more desirable as it saves time, travel and NHS resources. Overall, patients felt that if they had a postoperative complication they would have preferred to have seen a clinician in person. We found patients' views influenced by the COVID-19 pandemic meaning they saw additional benefits of telephone follow up compared to face-toface review.

Conclusions The majority of patients felt that TFU after cataract surgery was an acceptable way to receive healthcare. This has implications for how follow up is provided after the most common operation in the UK and worldwide and opens the possibility of this being provided by an automated AI driven system in the future.

\section{REDUCING INTRA-HOSPITAL TELEPHONE COMMUNICATION TIME USING APP TECHNOLOGY}

Mohammed Hamid. Royal Shrewsbury Hospital, UK

10.1136/leader-2020-FMLM.111

Background Lengthy switchboard waiting times result in delayed communication between healthcare professionals in a hospital. Wasted time impedes patient care, costs the Trust a substantial financial sum and impacts healthcare professional's morale. Aim: To reduce intra-hospital telecommunication time utilising the Induction phone application, an easy to use, regularly updated telephone directory. Methods: Initial audit: Five chosen specialities were contacted between 9-10 AM from the Emergency department for 2 consecutive weeks. The time taken to reach each speciality via switchboard was recorded. A survey seeking the number of calls made per day, the preferred method of contact and the feelings associated with telephone waiting times was sent to department doctors. PDSA cycle 1: One-month application advertisement and re-audit. PDSA2: Eye-catching tele-directory board with the most used extensions and bleeps displayed in the department. Satisfaction survey sent post PDSA2. Sustainability: New doctors were provided induction information. Results: Initial average waiting time via switchboard was 48 seconds. The average calls made per doctor each day was 12 . This calculated to a total departmental loss of 20.16 hours per week waiting on the phone, equating an annual loss of $£ 26,208$. PDSA1: Average waiting times reduced to 12 seconds utilising the application; saving an estimate $\sim £ 19,656$ per annum. PDSA2: Instant availability of contact details on the display board further reduced waiting times to an average 6 seconds. $84 \%$ of doctors $(n=16)$ disliked waiting more than 20 seconds, with associated feelings of frustration. $100 \%$ preferred the display board, then the use of the application before resorting to switchboard. 100\% Sustainability was recorded one year later. Conclusion: The use of application technology reduces wasted time which hampers patient care; reduces Trust running costs; and improves health care professional's morale at work.

\section{Understanding leadership through research}

\section{VILLAGES AGAINST CERVICAL CANCER: IMPROVING INDIA'S CERVICAL CANCER BURDEN BY EMPOWERING ASHA WORKERS}

1,2Sarayoo Ravishankar Vaidya. 'Independent researcher, MS Ramaiah Medical College Bengaluru, India; ${ }^{2}$ Masters in International Health student, Charité - Universitätsmedizin Berlin, Germany

\subsection{6/leader-2020-FMLM.112}

Cervical cancer is the second leading cause of new cancer cases and cancer-related deaths among women in India, with an estimated 96,922 new cases and 60,078 deaths each year. (ICCP,2020) India bears over a tenth of the global burden of cancers, with preventable cancers like cervical, oral and breast cancer forming more than a third of the disease burden.(Ferlay, 2015).

Currently, early diagnosis via low cost screening methods play the biggest role in curbing the burden of disease. The screening methods employed are colposcopy examination and routine pap smears every 5 years. However, both screening methods, while effective in identifying the disease, require skilled clinicians and laboratories with appropriate set-ups.

One method to circumvent this problem of low screening rates is to train rural local women to carry out Visual Inspection of cervix with Acetic acid (VIA) as a primary method of screening, as it is a low cost method of screening which does not require much laboratory facilities.

The aim is to scale up the current rate of screening of cervical cancer and subsequently decrease the case fatality rate of cervical cancer in Indian villages. This will be done by training Female health care workers and rural health practitioners to carry out Visual inspection of the Cervix.

Overall, a lot is left to be done to help improve the burden of cancer in India, but the best route is to start with harmony of Public and private sector and prioritization of cancer care in India.

There is also a need to improve connectivity between rural and urban areas to facilitate shunting of cancer patients for tertiary care. Coordination with Ministries of urban development, transportation and infrastructure developers is necessary to ensure this.

\section{Leadership lessons from across the world}

\section{TRAINING EMERGENCY MEDICAL STAFF TO APPROPRIATELY HANDLE PATIENTS WITH CHEST PAIN}

\footnotetext{
1,2Sarayoo Ravishankar Vaidya. 'Independent researcher, MS Ramaiah Medical College Bengaluru, India; ${ }^{2}$ Masters in International Health student, Charité - Universitätsmedizin Berlin, Germany
}

\subsection{6/leader-2020-FMLM.113}

Coronary artery disease(CAD) is a common cause of morbidity and mortality in the world and many patients with CAD present with chest pain in an emergency setting as a first presentation. In emergency settings in India, the burden of diagnosis and treatment of patients with chest pain often falls on the emergency physician alone, who also has to deal with 